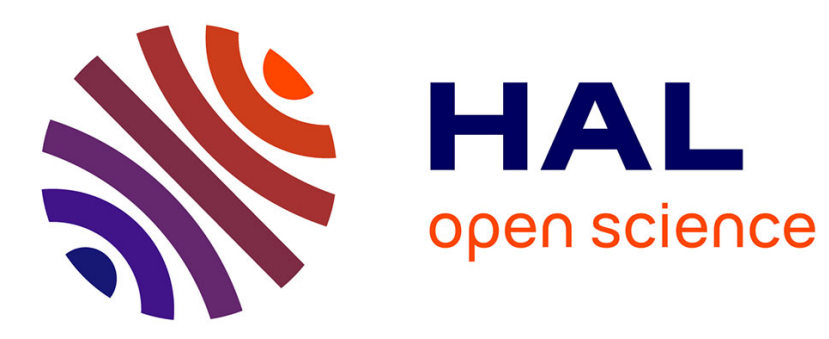

\title{
Regional Stabilization of Input-Delayed Uncertain Nonlinear Polynomial Systems
}

Daniel Coutinho, Carlos E de Souza, João Manoel Gomes da Silva, Andre Caldeira, Christophe Prieur

\section{- To cite this version:}

Daniel Coutinho, Carlos E de Souza, João Manoel Gomes da Silva, Andre Caldeira, Christophe Prieur. Regional Stabilization of Input-Delayed Uncertain Nonlinear Polynomial Systems. IEEE Transactions on Automatic Control, 2020, 65 (5), pp.2300-2307. 10.1109/TAC.2019.2931952 . hal-02917930

\section{HAL Id: hal-02917930 \\ https://hal.science/hal-02917930}

Submitted on 20 Aug 2020

HAL is a multi-disciplinary open access archive for the deposit and dissemination of scientific research documents, whether they are published or not. The documents may come from teaching and research institutions in France or abroad, or from public or private research centers.
L'archive ouverte pluridisciplinaire HAL, est destinée au dépôt et à la diffusion de documents scientifiques de niveau recherche, publiés ou non, émanant des établissements d'enseignement et de recherche français ou étrangers, des laboratoires publics ou privés. 


\title{
Regional Stabilization of Input-Delayed Uncertain Nonlinear Polynomial Systems
}

\author{
Daniel Coutinho, Carlos E. de Souza, João M. Gomes da Silva Jr, André F. Caldeira and Christophe Prieur
}

\begin{abstract}
This paper addresses the problem of local stabilization of nonlinear polynomial control systems subject to time-varying input delay and polytopic parameter uncertainty. A linear matrix inequality approach based on the LyapunovKrasovskii theory is proposed for designing a nonlinear polynomial state feedback controller ensuring the robust local uniform asymptotic stability of the system origin along with an estimate of its region of attraction. Two convex optimization procedures are presented to compute a stabilizing controller ensuring either a maximized set of admissible initial states for given upper bounds on the delay and its variation rate or a maximized lower bound on the maximum admissible input delay considering a given set of admissible initial states. Numerical examples demonstrate the potentials of the proposed stabilization approach.
\end{abstract}

Index Terms-Nonlinear polynomial systems, time-varying input delay, local stabilization, region of attraction estimation.

\section{INTRODUCTION}

The stability analysis and control synthesis of dynamical systems under delayed inputs is a problem that has been attracting the interest of control practitioners over the last decades due to the inherent transport delays in practical applications, and more recently because of induced communication delays in the context of networked control systems (see, for instance, [1]-[4] and references therein). For linear systems, there exist several approaches to deal with input delay in the frequency and state-space domains such as the predictorbased approach [5], the model reduction technique [6], and the ones employing either Lyapunov-Razumikhin or LyapunovKrasovskii stability theories to take model uncertainties and varying delays into account - see, e.g., [7], [8] and the references therein.

In the context of nonlinear systems, results dealing with model uncertainties and/or time-varying input delays have been proposed in the literature. For instance, [9] addresses

This work was partially supported by the CAPES/Brazil and CNPq/Brazil under grants 478018/2013-4, 422992/2016-0, 305979/2015-9/PQ, 30.8949/2016-1/PQ and 304351/2014-8/PQ.

Daniel Coutinho is with the Department of Automation and Systems, UFSC, PO BOX 476, 88040 - 900, Florianópolis, SC, Brazil coutinho@das.ufsc.br

Carlos E. de Souza is with the Department of Mathematical and Computational Methods, Laboratório Nacional de Computação Científica (LNCC/MCTIC), Petrópolis, RJ 25651-075, Brazil. c souza@ lncC.br

João M. Gomes da Silva Junior is with Department of Automation and Energy Systems, Universidade Federal do Rio Grande do Sul (UFRGS), Porto Alegre, RS, Brazil. jmgomes@ufrgs.br

André F. Caldeira is with the Graduate Program on Engineering of Automation and Systems (PPGEAS), Universidade Federal de Santa Catarina (UFSC), PO BOX 476, 88040 - 900, Florianópolis, SC, Brazil and was with Gipsa-lab, Grenoble, France. caldeiraaf@gmail.com

Christophe Prieur is with Université Grenoble Alpes, CNRS, Grenoble INP, Gipsa-lab, France. christophe.prieurdgipsa-lab. fr the input-to-state stability problem for the class of input affine nonlinear systems subject to a constant input delay, [10] considers the stabilization problem of a chain of integrators with nonlinearities subject to norm-bounded uncertainties and input delay, [11] focuses on the stabilization of uncertain EulerLagrange systems with a time-varying input delay, [12] studies the stability properties of a class of switched nonlinear systems subject to input delays, and [13] proposes a Smith predictor like input delay compensation for nonlinear systems in either forward complete or strict-feedforward form. An alternative approach to deal with input-delayed nonlinear systems is to consider a linear approximation of the system around an operating point (e.g., obtained via the Taylor expansion), and then apply well-established control design tools for linear input-delayed systems. If the error introduced by a linear approximation is too large, high-order terms of the Taylor expansion can be considered to more accurately describe the system dynamics leading to nonlinear polynomial models. Besides, many practical systems are exactly described by polynomial systems such as heating columns [14], DC-DC converters [15], aircraft systems [16], and unmanned aerial vehicles [17]. Due to the good compromise between model accuracy and mathematical complexity, the class of nonlinear polynomial systems has been in the last ten or more years the subject of intensive research generally using either the sum-of-squares technique [18], [19], or the multiplier-based methods [20], [21]. In particular, local and global stability analysis problems of state-delayed polynomial systems have been addressed in [22], [23] and [24].

Inspired by the delay-dependent method proposed in [7] to deal with the stabilization of linear systems with input delay and actuator saturation, this paper investigates the local stabilization of nonlinear polynomial systems with a time-varying input delay and polytopic parameter uncertainty considering a Lyapunov-Krasovskii functional. Note that the stabilization of input-delayed nonlinear systems which are open-loop unstable and only locally stabilizable is more involved than the linear system counterpart as it is required to guarantee that the system state trajectory in the initial time interval before any control signal is applied does not leave the region of attraction of the system equilibrium to be stabilized [7]. Motivated by this fact, a linear matrix inequality (LMI) based method is proposed in this paper for designing a nonlinear polynomial state feedback controller ensuring the robust local uniform asymptotic stability of the system zero equilibrium point while providing an estimate of its region of attraction. In addition, thanks to a novel polynomial decomposition of the system dynamics, two convex optimization procedures are presented for 
designing a stabilizing controller ensuring either a maximized set of admissible initial states for a given maximum delay and its variation rate, or a maximized lower bound on the maximum admissible input delay considering a given region of admissible initial states.

This paper is organized as follows. Section II introduces the class of input-delayed systems considered in this paper and the stabilization problem to be addressed, whereas Section III presents a key result which will be instrumental to the controller design. Then, the main result of this paper is derived in Section IV along with two convex optimization procedures to design a controller that provides either a maximized set of admissible initial states or a maximized lower bound on the maximum admissible delay. Numerical examples are given in Section V and Section VI provides some concluding remarks.

Notation: $\mathbb{R}$ is the set of real numbers, $\mathbb{R}^{n}$ is the $n$ dimensional Euclidean space, $\mathbb{R}^{m \times n}$ is the set of $m \times n$ real matrices, $I_{n}$ in the $n \times n$ identity matrix, $0_{n}$ and $0_{n \times m}$ are respectively the $n \times n$ and $n \times m$ matrices of zeros, and $\operatorname{diag}\{\cdots\}$ denotes a block-diagonal matrix. For a real matrix $S, S^{\prime}$ denotes its transpose, $\operatorname{He}\{S\}$ stands for $S+S^{\prime}$, and $S>0$ means that $S$ is symmetric and positivedefinite. For two polytopes $\mathcal{A}$ and $\mathcal{B}, \mathcal{A} \times \mathcal{B}$ denotes the meta-polytope obtained by the cartesian product and $\mathcal{V}(\mathcal{A})$ is the set of all the vertices of $\mathcal{A}$. For a vector $\xi \in \mathbb{R}^{n}$, $R: \mathbb{R}^{n} \rightarrow \mathbb{R}^{m \times q}$ is an affine matrix function of $\xi$ if it can be cast as $R(\xi)=R_{0}+\sum_{i=1}^{n} \xi_{i} R_{i}$, where $R_{i} \in \mathbb{R}^{m \times q}$, $i=0,1, \ldots, n$, and $\xi_{i}$ is the $i$-th element of $\xi$. The Banach space of continuous functions $\phi:[a, b] \rightarrow \mathbb{R}^{n}$ with finite norm $\|\phi\|_{[a, b]}:=\sup _{a \leq t \leq b}\|\phi(t)\|$ is denoted by $\mathcal{C}_{[a, b]}^{n}$, where $\|\cdot\|$ is the Euclidean vector norm, and $x_{t} \in \mathcal{C}_{[-d, 0]}^{n}$ is a segment of the function $x_{t}(s)=x(t+s), \forall s \in[-d, 0]$.

\section{Problem StATEMENT}

Consider the following class of input-delayed nonlinear control systems:

$$
\left\{\begin{array}{l}
\dot{x}(t)=f(x(t), \delta)+B(x(t), \delta) u(t-\tau(t)), \forall t \geq 0, \\
x(0)=\bar{x}_{0}, \\
u(t)=K(x(t)) x(t), \forall t \geq 0,
\end{array}\right.
$$

where $x(t) \in \mathcal{X} \subset \mathbb{R}^{n}$ is the state, $u(t) \in \mathbb{R}^{n_{u}}$ is the control input, $\delta \in \Delta \subset \mathbb{R}^{n_{\delta}}$ is a vector of uncertain constant parameters, $f(x, \delta): \mathcal{X} \times \Delta \rightarrow \mathbb{R}^{n}$ and $B(x, \delta): \mathcal{X} \times \Delta \rightarrow \mathbb{R}^{n \times n_{u}}$ are polynomial functions of $x$ and affine with respect to $\delta$, with $f(0, \delta)=0, \forall \delta \in \Delta, K(x): \mathcal{X} \rightarrow \mathbb{R}^{n_{u} \times n}$ is a polynomial function of $x$ to be determined, $\bar{x}_{0}$ is the initial state, and $\tau(t) \in \mathbb{R}$ is a time-varying input delay satisfying:

$$
0 \leq \tau(t) \leq d, \quad \dot{\tau}(t) \leq h \leq 1, \quad \forall t \geq 0,
$$

with $d$ and $h$ being given positive scalars. $\mathcal{X}$ and $\Delta$ are compact regions defining respectively the state and uncertainty domains with $\mathcal{X}$ containing $x=0$. In addition, similarly as in [7], it is assumed that the controller is turned on at $t=0$ and $u(t)=0, \forall t<0$. For $t \geq 0$, due to the input delay, the control signal is computed from the past state information whenever $t-\tau(t) \geq 0$, otherwise the control input is set to zero. Note that this control setting is effectively a practical problem and appears in a number of control applications as, for instance, in sampled-data control systems with transmission delays.

In view of (2), there exists a unique $t_{0} \leq d$ such that $t-\tau(t)<0, \forall t \in\left[0, t_{0}\right)$, and $t-\tau(t) \geq 0, \forall t \geq t_{0}$. As a consequence, since $u(t-\tau(t))=0$ for $t \in\left[0, t_{0}\right)$, the system operates in open loop in the time interval $\left[0, t_{0}\right)$. Hence, the controlled system can be cast as follows:

$$
\begin{gathered}
\dot{x}(t)= \begin{cases}f(x(t), \delta), & \text { for } 0 \leq t<t_{0}, \\
f_{c l}(x(t), \tilde{x}(t), \delta), & \text { for } t \geq t_{0},\end{cases} \\
\left\{\begin{array}{l}
f_{c l}(x(t), \tilde{x}(t), \delta)=f(x(t), \delta)+B(x(t), \delta) K(\tilde{x}(t)) \tilde{x}(t), \\
x(0)=\bar{x}_{0}, \\
\tilde{x}(t)=x(t-\tau(t)) .
\end{array}\right.
\end{gathered}
$$

Note that $x=0$ is an equilibrium solution of system (3) and in this paper attention will be focused on stability properties of this equilibrium. For notation simplicity, hereafter the argument $t$ of $x(t), \tilde{x}(t), u(t)$ and $\tau(t)$ will be often omitted.

It should be observed that since in the time interval $\left[0, t_{0}\right)$ system (3) operates in open-loop, it is of fundamental importance in the stabilization problem to explicitly take into account the system behavior over the latter interval when the equilibrium $x=0$ of the open-loop system of (1) is unstable. In this case, the state trajectory starting at some initial value $\bar{x}_{0}$ will move away from the equilibrium point until the feedback signal starts to be applied to the system at $t=t_{0}$ and thus it may leave the region of attraction of the closed-loop system origin before any control action is actually applied to the system. Thus, the delayed state feedback will not be able to asymptotically stabilize the system origin. In order to prevent this situation and to properly address the system closed-loop stability behavior in the vicinity of the zero equilibrium solution, it will be considered in this paper the following definition of robust regional stability.

Definition 1: Consider the system in (1) for a given stabilizing state feedback and let $\mathcal{R}_{0}$ and $\mathcal{R}$ be two compact sets such that $\mathcal{R}_{0} \subset \mathcal{R} \subset \mathcal{X}$ with $\mathcal{R}_{0}$ containing $x=0$. The equilibrium point $x=0$ of system of (1) is robustly regionally stable if, for any $\bar{x}_{0} \in \mathcal{R}_{0}$ and all $\delta \in \Delta$, the state trajectory $x(t)$ remains confined to $\mathcal{R}$, for all $t \geq 0$ and $x(t) \rightarrow 0$ as $t \rightarrow \infty$.

In light of the above scenario, this paper is concerned with obtaining a numerical and tractable solution to the robust regional stabilization problem for uncertain input-delayed nonlinear polynomial systems. More precisely, this paper is focused on the design of a polynomial state feedback control law $u=K(x) x$ ensuring the robust regional stability of the control system in (1) while achieving either: $(i)$ a maximized set of initial states $\mathcal{R}_{0}$ for a given maximum delay $d$; or $(i i)$ a maximized lower bound on the maximum admissible delay $d$ for a given set $\mathcal{R}_{0}$ of admissible initial states.

Before ending this section, the following two lemmas, that will be instrumental to derive the main results of this paper, are recalled from the literature.

Lemma 1 ([25]): Let $P$ and $G$ be $n \times n$ real matrices with $P>0$ and $G$ nonsingular. Then, the following holds:

$$
P^{-1} \geq G+G^{\prime}-G^{\prime} P G \text {. }
$$


Lemma 2 ([26]): For any $n \times n$ real matrix $Q>0$, real scalars $d_{1}$ and $d_{2}$ such that $d_{1}<d_{2}$, and any continuous function $w:\left[d_{1}, d_{2}\right] \mapsto \mathbb{R}^{n}$, the following holds:

$$
\begin{aligned}
\left(d_{2}-d_{1}\right) \int_{d_{1}}^{d_{2}} w(s)^{\prime} Q w(s) d s \geq & \\
& {\left[\int_{d_{1}}^{d_{2}} w(s) d s\right]^{\prime} Q\left[\int_{d_{1}}^{d_{2}} w(s) d s\right] . }
\end{aligned}
$$

\section{PREliminary Results}

This section presents an extension of the solution bound approach proposed in [7] for input-delayed linear systems via the Lyapunov-Krasovskii method to the context of general nonlinear systems with input delay. This result provides the means to derive an LMI-based solution to the robust regional stabilization problem as stated in Section II. Firstly, a local stability result for retarded functional differential equations is recalled. To this end, consider the following delayed statespace representation:

$$
\left\{\begin{array}{l}
\dot{x}=g\left(t, x_{t}\right), \forall t \geq t_{i}, \quad x \in \mathbb{R}^{n}, \\
x_{t_{i}}=\psi \in \mathcal{C}_{[-d, 0]}^{n} .
\end{array}\right.
$$

It is assumed that $g: \mathbb{R} \times \mathcal{C}_{[-d, 0]}^{n} \mapsto \mathbb{R}^{n}$, with $g(t, 0)=0$, $\forall t \geq t_{i}$, satisfies the conditions of existence and uniqueness of solution for any $t \geq t_{i}$. Next, for system (7), we present the following local version of the Lyapunov-Krasovskii stability theorem [27].

Lemma 3: Let $u, v, w: \mathbb{R} \mapsto \mathbb{R}$ be continuous nondecreasing and positive definite functions, $\mathcal{X} \in \mathbb{R}^{n}$ a bounded compact set containing $x=0$, and

$$
\mathcal{X}_{a}:=\left\{\phi \in \mathcal{C}_{[-d, 0]}^{n}: \phi(s) \in \mathcal{X}, \forall s \in[-d, 0]\right\} .
$$

Suppose there exists a continuously differentiable functional $V: \mathbb{R} \times \mathcal{C}_{[-d, 0]}^{n} \mapsto \mathbb{R}^{+}$satisfying

$$
\begin{aligned}
& u(\|x(t)\|) \leq V\left(t, x_{t}\right) \leq v\left(\left\|x_{t}\right\|_{[-d, 0]}\right), \forall x_{t} \in \mathcal{X}_{a}, t \geq t_{i}, \\
& \dot{V}\left(t, x_{t}\right)<-w(\|x(t)\|), \forall x_{t} \in \mathcal{X}_{a}, t \geq t_{i},
\end{aligned}
$$

where $\dot{V}\left(t, x_{t}\right)$ denotes the time-derivative of $V\left(t, x_{t}\right)$ along the solution of (7). Then, the equilibrium solution $x=0$ of (7) is uniformly asymptotically stable.

Since the delayed input is a function of the state, note that the closed-loop system in (3) is of the form of system (7). However, when the zero equilibrium of the open-loop system of (1) is unstable, Lemma 3 cannot be applied to system (3) for all $t \geq 0$ because there is no $V\left(t, x_{t}\right)$ satisfying the second condition in (9). Indeed, if such a $V\left(t, x_{t}\right)$ existed and since $f(\cdot)$ is time-invariant, the zero equilibrium of the system $\dot{x}=f(x, \delta)$ would be locally asymptotically stable, which is a contradiction. To overcome this difficulty, an approach inspired by [7] is presented in the sequel. The idea consists on ensuring that the state trajectory in the interval $\left[0, t_{0}\right)$ does not leave the region of attraction of the zero equilibrium solution of the closed-loop system and then to apply Lemma 3 to system (3) for all $t \geq t_{0}$. To this end, let the following LyapunovKrasovskii functional candidate:

where

$$
V\left(t, x_{t}, \dot{x}_{t}\right)=V_{1}(x(t))+V_{2}\left(t, x_{t}\right)+V_{3}\left(\dot{x}_{t}\right),
$$

$$
\begin{aligned}
& V_{1}(x(t))=x(t)^{\prime} P_{1} x(t), \\
& V_{2}\left(t, x_{t}\right)=\int_{t-\tau(t)}^{t} x(\alpha)^{\prime} P_{21} x(\alpha) d \alpha+\int_{t-d}^{t} x(\alpha)^{\prime} P_{22} x(\alpha) d \alpha, \\
& V_{3}\left(\dot{x}_{t}\right)=d \int_{-d}^{0} \int_{t+\beta}^{t} \dot{x}(\alpha)^{\prime} P_{3} \dot{x}(\alpha) d \alpha d \beta
\end{aligned}
$$

with $P_{1}, P_{21}, P_{22}$ and $P_{3}$ being symmetric positive definite matrices. To simplify the notation, let $V(t):=V\left(t, x_{t}, \dot{x}_{t}\right)$.

The next lemma gives conditions to ensure the robust regional stability of system (1).

Lemma 4: Consider system (1) with $f(\cdot), B(\cdot)$ and $K(\cdot)$ being general nonlinear functions satisfying the conditions of existence and uniqueness of solution for any $t \geq 0$, the Lyapunov-Krasovskii functional given in (10) and the following sets:

$$
\mathcal{R}_{0}=\left\{x \in \mathbb{R}^{n}: x^{\prime} P_{1} x \leq 1\right\}, \mathcal{R}=\left\{x \in \mathbb{R}^{n}: x^{\prime} P_{1} x \leq \gamma\right\},
$$

with $\gamma>0$ as defined in the sequel. Let $\dot{V}\left(t, x_{t}, \dot{x}_{t}, \delta\right)$ and $\dot{V}_{1}(x(t), \delta)$ be respectively the time-derivatives of $V\left(t, x_{t}, \dot{x}_{t}\right)$ and $V_{1}(x(t))$ along the trajectories of (1). Suppose there exist positive scalars $\epsilon, \sigma$ and $\varphi$ satisfying the following conditions: C1 $\dot{V}\left(t, x_{t}, \dot{x}_{t}, \delta\right) \leq-\epsilon\|x(t)\|^{2}, \forall x_{t} \in \mathcal{X}_{a}, t \geq t_{0}, \forall \delta \in \Delta$; C2 $\dot{V}_{1}(x(t), \delta)-2 \varphi V_{1}(x(t)) \leq 0, \forall x(t) \in \mathcal{X}, t \in\left[0, t_{0}\right)$, $\forall \delta \in \Delta$

C3 $\dot{V}\left(t, x_{t}, \dot{x}_{t}, \delta\right)-2 \varphi V_{1}(x(t)) \leq 0, \forall x_{t} \in \mathcal{X}_{a}, t \in\left[0, t_{0}\right)$, $\forall \delta \in \Delta$;

C4 $\sigma P_{1}-d\left(P_{21}+P_{22}\right) \geq 0$;

C5 $\mathcal{R} \subset \mathcal{X}$, with $\gamma=\sigma+\mathrm{e}^{2 \varphi d}$.

Then, provided that $\bar{x}_{0} \in \mathcal{R}_{0}$, the state trajectory $x(t)$, for all $t \geq 0$ and $\delta \in \Delta$, will never leave $\mathcal{R}$ and $x(t) \rightarrow 0$ as $t \rightarrow \infty$.

Proof: Notice from Lemma 3 that $\mathbf{C} \mathbf{1}$ implies the asymptotic convergence to zero of $x(t)$ provided that the state trajectory $x(t)$, for any $t \in\left[0, t_{0}\right)$, is confined to a region $\tilde{\mathcal{R}}(\gamma):=\left\{x_{t} \in \mathcal{C}_{[-d, 0)}^{n}: V\left(t, x_{t}, \dot{x}_{t}\right) \leq \gamma\right\} \subset \mathcal{X}_{a}$, for some $\gamma>0$. It will be shown in the sequel that $\mathbf{C 2}$ and $\mathbf{C 3}$ guarantee for all $t \in\left[0, t_{0}\right)$ that $x(t)$ lies inside the region $\mathcal{R} \subset \mathcal{X}$ and $V\left(t, x_{t}, \dot{x}_{t}\right) \leq \gamma$, with $\gamma$ as in $\mathbf{C 5}$, provided that $\bar{x}_{0}$ belongs to the set $\mathcal{R}_{0}$.

Firstly, by [28, Lemma 2.5], C2 implies the following:

$$
V_{1}(x(t)) \leq \mathrm{e}^{2 \varphi t} V_{1}(x(0)), \forall t \in\left[0, t_{0}\right), \delta \in \Delta .
$$

Integrating $\mathbf{C} 3$ from 0 to $t$, for any $t \in\left[0, t_{0}\right)$, leads to

$V(t) \leq V(0)+2 \varphi \int_{0}^{t} V_{1}(x(s)) d s, \forall t \in\left[0, t_{0}\right), \delta \in \Delta$,

which together with (13) yields:

$$
V(t) \leq V(0)+V_{1}(x(0))\left(\mathrm{e}^{2 \varphi t}-1\right), \forall t \in\left[0, t_{0}\right), \delta \in \Delta .
$$

Next, an upper bound on $V(0)$ is derived from (10) using arguments similar to those as in [7]. To this end, since the solution to (3) for $t \in\left[0, t_{0}\right)$ does not depend on the value of $x(s)$ for $s<0$, similar as in [7] it is considered that $x(s)=$ $\bar{x}_{0}, \forall s \in[-d, 0]$. Hence, the following is obtained from (10) and (11):

$$
\begin{aligned}
V(0) & =\bar{x}_{0}^{\prime} P_{1} \bar{x}_{0}+\int_{-\tau(0)}^{0} \bar{x}_{0}^{\prime} P_{21} \bar{x}_{0} d \alpha+\int_{-d}^{0} \bar{x}_{0}^{\prime} P_{22} \bar{x}_{0} d \alpha \\
& =\bar{x}_{0}^{\prime}\left(P_{1}+\tau(0) P_{21}+d P_{22}\right) \bar{x}_{0} \\
& \leq \bar{x}_{0}^{\prime}\left[P_{1}+d\left(P_{21}+P_{22}\right)\right] \bar{x}_{0}
\end{aligned}
$$

As $V_{1}(x(0))=\bar{x}_{0}^{\prime} P_{1} \bar{x}_{0}$ and taking (16) and $\mathbf{C 4}$ into account, (15) leads to:

$$
V(t) \leq\left(\sigma+\mathrm{e}^{2 \varphi t}\right) \bar{x}_{0}^{\prime} P_{1} \bar{x}_{0}, \forall t \in\left[0, t_{0}\right), \delta \in \Delta .
$$

Hence, if $\bar{x}_{0} \in \mathcal{R}_{0}$ and since $t_{0} \leq d$, the following holds:

$$
V(t) \leq \gamma, \forall t \in\left[0, t_{0}\right), \delta \in \Delta ; \text { with } \gamma=\sigma+\mathrm{e}^{2 \varphi d} \text {. }
$$


Next, considering that by (10) we have $V(t) \geq V_{1}(x(t))$, it follows that:

$$
V_{1}(x(t)) \leq V(t) \leq \gamma, \forall t \in\left[0, t_{0}\right), \delta \in \Delta .
$$

Thus, provided that $\bar{x}_{0} \in \mathcal{R}_{0}$, the inequality in (18) ensures that $x(t) \in \mathcal{R}$ for all $t \in\left[0, t_{0}\right)$ and $\delta \in \Delta$. In addition, if C1 holds, then $\dot{V}(t)<0$, for all $t \geq t_{0}$ and $\delta \in \Delta$, and hence $V_{1}(x(t)) \leq V(t) \leq V\left(t_{0}\right) \leq \gamma, \forall t \in\left[t_{0}, \infty\right), \delta \in \Delta$. Finally, in view of $\mathbf{C 5}$, the state trajectory $x(t)$, for all $t \geq 0$ and $\delta \in \Delta$, will never leave $\mathcal{R}$ and thus $x_{t} \in \mathcal{X}_{a}, \forall t \geq t_{0}$, which from Lemma 3 ensures that $x(t) \rightarrow 0$ as $t \rightarrow \infty$.

Remark 1: It should be pointed out that the setting considered in this paper differs from the one proposed, e.g., in [1], where it is assumed that $u(s)$, for $s \in[-d, 0]$, is defined by an initial actuator trajectory $\phi(s), s \in[-d, 0]$. In this case, $u(t-\tau(t))$ will also affect the system dynamics over the interval $\left[0, t_{0}\right)$. Precisely, the system dynamics for $t \in\left[0, t_{0}\right)$ will be $\dot{x}(t)=f(x(t), \delta)+B(x(t), \delta) w(t)$, with $w(t)=u(t-\tau(t))$, $u(s)=\phi(s), s \in[-d, 0]$. Then, to obtain a bound on the system trajectory in this interval, we have to modify conditions $\mathbf{C 2}$ and $\mathbf{C 3}$ and the definition of $\gamma$ in C5 of Lemma 4 accordingly. For instance, assuming that $\phi:[-d, 0] \rightarrow \mathbb{R}^{n_{u}}$ is a continuously differentiable function with a finite norm $\|\phi\|_{d}=$ $\sup _{-d \leq t \leq 0}\|\phi(t)\| \leq \lambda, \mathbf{C} 2$ and $\mathbf{C 3}$ can be respectively modified as: (C2') $\dot{V}_{1}(x(t), \delta)-2 \varphi V_{1}(x(t))-\kappa w(t)^{\prime} w(t)<0$, $\forall x \in \mathcal{X}, t \in\left[0, t_{0}\right), \delta \in \Delta, w \in \mathcal{W}$ and $\kappa>0$; and (C3') $\dot{V}\left(t, x_{t}, \dot{x}_{t}, \delta\right)-2 \varphi V_{1}(x(t))-w(t)^{\prime} w(t)<0, \forall x_{t} \in \mathcal{X}_{a}$, $t \in\left[0, t_{0}\right), \delta \in \Delta$ and $w \in \mathcal{W}$, where $\mathcal{W}=\left\{w \in \mathbb{R}^{n_{u}}\right.$ : $\left.w(t)^{\prime} w(t) \leq \lambda^{2}\right\}$. However, due to space limitation, this case is not addressed in this paper.

\section{Main Results}

This section starts by introducing the following polynomial decomposition of the vector and matrix functions $f(x, \delta)$ and $B(x, \delta)$ :

$$
\left\{\begin{array}{l}
f(x, \delta)=\Pi_{a}(x)^{\prime} A_{a}(\delta) x, B(x, \delta)=\Pi_{a}(x)^{\prime} B_{a}(\delta), \\
\Pi_{a}(x)=\left[\begin{array}{ll}
I_{n} & \Pi(x)^{\prime}
\end{array}\right]^{\prime},
\end{array}\right.
$$

where $A_{a}: \Delta \mapsto \mathbb{R}^{m \times n}$ and $B_{a}: \Delta \mapsto \mathbb{R}^{m \times n_{u}}$ are affine matrix functions of $\delta$, and $\Pi: \mathcal{X} \mapsto \mathbb{R}^{(m-n) \times n}$ is a polynomial function of $x$ such that the following constraint holds:

$$
\Omega_{1}(x)+\Omega_{2}(x) \Pi(x)=0,
$$

for some affine matrix functions $\Omega_{1}: \mathcal{X} \mapsto \mathbb{R}^{q \times n}$ and $\Omega_{2}: \mathcal{X} \mapsto \mathbb{R}^{q \times(m-n)}, q \geq m-n$. Notice that the matrix $\Pi(x)$ in the decomposition in (19) and (20) contains monomials in $x$ of $f(x, \delta)$ of degree equal or larger than one and monomials in $x$ of $B(x, \delta)$ not appearing in $f(x, \delta)$ (with sum of monomials being also allowed). Observe that the integer $m$ will be determined by the choice of monomials in $x$ comprised in $\Pi(x)$.

It is assumed that the matrix $\Omega_{2}(x)$ is full column-rank for all $x \in \mathcal{X}$. It should be noticed that this assumption does not imply any loss of generality because it is always possible to choose a polynomial matrix $\Pi(x)$ ensuring that such an assumption holds.

Remark 2: It can be easily verified using standard polynomial manipulations that there always exists a polynomial decomposition as in (19) and (20) by a suitable choice of $\Pi(x)$ [21]. The reader may follow the numerical examples in Section V for details on how to choose the matrix $\Pi(x)$.

Next, taking the Lyapunov-Krasovskii functional in (10) into account, the time-derivative of $V(t)$, denoted by $\dot{V}(t)$, is given by:

$$
\begin{array}{r}
\dot{V}(t)=2 x^{\prime} P_{1} \dot{x}+x^{\prime}\left(P_{21}+P_{22}\right) x-(1-\dot{\tau}) \tilde{x}^{\prime} P_{21} \tilde{x} \\
-\breve{x}^{\prime} P_{22} \breve{x}+d^{2} \dot{x}^{\prime} P_{3} \dot{x}-d \int_{t-\tau(t)}^{t} \dot{x}^{\prime}(\alpha) P_{3} \dot{x}(\alpha) d \alpha \\
+d \int_{t-d}^{t-\tau(t)} \dot{x}^{\prime}(\alpha) P_{3} \dot{x}(\alpha) d \alpha,
\end{array}
$$

where $\tilde{x}$ is as in (4) and

$$
\breve{x}:=x(t-d) .
$$

In view of Lemma 2 and since $\dot{\tau} \leq h$, the following upper bound on $\dot{V}(t)$ holds:

$$
\begin{aligned}
\dot{V}(t) \leq & 2 x^{\prime} P_{1} \dot{x}+x^{\prime}\left(P_{21}+P_{22}\right) x \\
& -(1-h) \tilde{x}^{\prime} P_{21} \tilde{x}-\breve{x}^{\prime} P_{22} \breve{x}+d^{2} \dot{x}^{\prime} P_{3} \dot{x} \\
& -\left[\int_{t-\tau(t)}^{t} \dot{x}(\alpha) d \alpha\right]^{\prime} P_{3}\left[\int_{t-\tau(t)}^{t} \dot{x}(\alpha) d \alpha\right] \\
& -\left[\int_{t-d}^{t-\tau(t)} \dot{x}(\alpha) d \alpha\right]^{\prime} P_{3}\left[\int_{t-d}^{t-\tau(t)} \dot{x}(\alpha) d \alpha\right] .
\end{aligned}
$$

Now, let the following notation:

$$
\begin{gathered}
\eta\left(x_{t}\right)=\left[\begin{array}{lll}
x^{\prime}(t) P_{1} & \tilde{x}(t)^{\prime} P_{1} & \breve{x}(t)^{\prime} P_{1} \\
\left(\int_{t-\tau(t)}^{t} \dot{x}(\alpha) d \alpha\right)^{\prime} P_{1} & \left(\int_{t-d}^{t-\tau(t)} \dot{x}(\alpha) d \alpha\right)^{\prime} P_{1}
\end{array}\right]^{\prime}, \\
\Upsilon(x, \tilde{x}, \delta)=\left[\begin{array}{lll}
d \Pi_{a}(x)^{\prime} A_{a}(\delta) Q_{1} & d \Pi_{a}(x)^{\prime} B_{a}(\delta) K(\tilde{x}) Q_{1} \\
0_{n} & 0_{n} & 0_{n}
\end{array}\right], \\
Q_{1}=P_{1}^{-1}, Q_{21}=Q_{1} P_{21} Q_{1}, \\
Q_{22}=Q_{1} P_{22} Q_{1}, \\
Q_{3}=Q_{1} P_{3} Q_{1} .
\end{gathered}
$$

Then, considering $\dot{x}(t)$ for $t \geq t_{0}$ given in (3), it follows that (23) for $t \geq t_{0}$ becomes:

$$
\begin{array}{r}
\dot{V}(t) \leq \eta\left(x_{t}\right)^{\prime}\left[\Psi(x, \tilde{x}, \delta)+\Upsilon(x, \tilde{x}, \delta)^{\prime} P_{3} \Upsilon(x, \tilde{x}, \delta)\right] \eta\left(x_{t}\right), \\
\forall t \geq t_{0}, \quad(27)
\end{array}
$$

where $\Psi(x, \tilde{x}, \delta)=\left[\Psi_{i j}\right], i, j=1, \ldots, 5$, is a symmetric block matrix function whose nonzero blocks $\Psi_{i j}, i=1, \ldots, 5, j=$ $i, \ldots, 5$, are given by:

$$
\begin{aligned}
& \Psi_{11}=\operatorname{He}\left\{\Pi_{a}(x)^{\prime} A_{a}(\delta) Q_{1}\right\}+Q_{21}+Q_{22}, \\
& \Psi_{12}=\Pi_{a}(x)^{\prime} B_{a}(\delta) K(\tilde{x}) Q_{1}, \Psi_{22}=-(1-h) Q_{21} \\
& \Psi_{33}=-Q_{22}, \Psi_{44}=-Q_{3}, \quad \Psi_{55}=-Q_{3} .
\end{aligned}
$$

Notice that since

$$
\begin{aligned}
& P_{1}\left[x(t)-\tilde{x}(t)-\int_{t-\tau(t)}^{t} \dot{x}(\alpha) d \alpha\right]=0, \\
& P_{1}\left[\tilde{x}(t)-\breve{x}(t)-\int_{t-d}^{t-\tau(t)} \dot{x}(\alpha) d \alpha\right]=0,
\end{aligned}
$$

it can be easily verified that the vector $\eta\left(x_{t}\right)$ is such that

$$
\mathcal{I} \eta\left(x_{t}\right) \equiv 0, \quad \mathcal{I}=\left[\begin{array}{ccccc}
I_{n} & -I_{n} & 0_{n} & -I_{n} & 0_{n} \\
0_{n} & I_{n} & -I_{n} & 0_{n} & -I_{n}
\end{array}\right] .
$$

Then, for any free multiplier matrix $R(x, \tilde{x}, \delta)$ to be determined, the following holds:

$$
\begin{array}{r}
\dot{V}(t) \leq \eta^{\prime}\left(x_{t}\right)\left[\Psi(x, \tilde{x}, \delta)+\Upsilon(x, \tilde{x}, \delta)^{\prime} P_{3} \Upsilon(x, \tilde{x}, \delta)+\right. \\
\operatorname{He}\{R(x, \tilde{x}, \delta) \mathcal{I}\}] \eta\left(x_{t}\right), \forall t \geq t_{0} .
\end{array}
$$


Applying similar arguments used to derive (31) and since from (3), $\dot{x}(t)=f(x(t), \delta), \forall t \in\left[0, t_{0}\right)$, and $x(s)=$ $\bar{x}_{0}, \forall s \in[-d, 0]$, it follows that $\tilde{x}(t)=\breve{x}(t)=\bar{x}_{0}$, $\forall t \in\left[0, t_{0}\right)$. Hence, the term $\dot{V}(t)-2 \varphi V_{1}(x(t)), \forall t \in\left[0, t_{0}\right)$, satisfies:

$$
\begin{aligned}
\dot{V}(t)- & 2 \varphi V_{1}(x(t)) \leq \widetilde{\eta}\left(x_{t}\right)^{\prime}[\widetilde{\Psi}(x, \delta) \\
& \left.+\widetilde{\Upsilon}(x, \delta)^{\prime} P_{3} \widetilde{\Upsilon}(x, \delta)+\operatorname{He}\{S(x, \delta) \widetilde{\mathcal{I}}\}\right] \widetilde{\eta}\left(x_{t}\right),
\end{aligned}
$$

where $S(x, \delta)$ is a free matrix function to be determined and

$$
\begin{aligned}
\widetilde{\eta}\left(x_{t}\right) & =\left[\begin{array}{lll}
x^{\prime}(t) P_{1} & \bar{x}_{0}^{\prime} P_{1} & \left(\int_{t-\tau(t)}^{t} \dot{x}(\alpha) d \alpha\right)^{\prime} P_{1}
\end{array}\right]^{\prime}, \\
\widetilde{\Upsilon}(x, \delta) & =\left[\begin{array}{lll}
d \Pi_{a}(x)^{\prime} A_{a}(\delta) Q_{1} & 0_{n} & 0_{n}
\end{array}\right], \\
\widetilde{\mathcal{I}} & =\left[\begin{array}{lll}
I_{n} & -I_{n} & -I_{n}
\end{array}\right], \\
\widetilde{\Psi} & =\operatorname{diag}\left\{\operatorname{He}\left\{\Pi_{a}(x)^{\prime} A_{a}(\delta) Q_{1}\right\}+Q_{21}+Q_{22}-2 \varphi Q_{1},\right. \\
& \left.-(1-h) Q_{21}-Q_{22},-Q_{3}\right\} .
\end{aligned}
$$

In the sequel, it will be proposed a solution to the robust regional stabilization problem in terms of a finite set of LMIs to ensure that conditions $\mathbf{C 1}, \mathbf{C 2}, \mathbf{C 3}, \mathbf{C 4}$ and $\mathbf{C 5}$ hold. To this end, it is assumed that $\mathcal{X}$ and $\Delta$ are given polytopic regions with known vertices with $0 \in \mathcal{X}$. In addition, it is considered that $\mathcal{X}$ is either defined in terms of the convex hull of its vertices or in the following equivalent form:

$$
\mathcal{X}=\left\{x \in \mathbb{R}^{n}: c_{i}^{\prime} x \leq 1, i=1, \ldots, n_{f}\right\},
$$

with $c_{i} \in \mathbb{R}^{n}, i=1, \ldots, n_{f}$, defining the $n_{f}$ faces of $\mathcal{X}$.

Theorem 1: Consider system (1) satisfying (2) and the polynomial decomposition as defined in (19) and (20). Let $\mathcal{X}$ and $\Delta$ be given polytopic regions defining the state and uncertainty domains, respectively. Suppose that for given positive scalars $d, h, \sigma$ and $\varphi$ there exist real matrices $F$, $Q_{1}>0, Q_{21}>0, Q_{22}>0, Q_{3}>0$ and $L_{i}$, for $i=1,2,3$, and affine matrix functions $R_{i j}(x, \tilde{x}, \delta), i=1, \ldots, 5, j=1,2$, and $S_{k}(x, \delta), k=1,2,3$, all having appropriate dimensions and satisfying the following LMIs:

$$
\begin{gathered}
\Phi(x, \tilde{x}, \delta)+\operatorname{He}\left\{L_{1} \Omega_{b}(x, \tilde{x})\right\}<0, \\
\forall(x, \tilde{x}, \delta) \in \mathcal{V}(\mathcal{X} \times \mathcal{X} \times \Delta), \\
\operatorname{He}\left\{A_{a}(\delta) Q_{1} N+L_{2} \Omega_{a}(x)\right\}-2 \varphi N^{\prime} Q_{1} N \leq 0, \\
\forall(x, \delta) \in \mathcal{V}(\mathcal{X} \times \Delta), \\
\Lambda(x, \delta)+\operatorname{He}\left\{L_{3} \Omega_{c}(x)\right\} \leq 0, \quad \forall(x, \delta) \in \mathcal{V}(\mathcal{X} \times \Delta), \\
\sigma Q_{1}-d\left(Q_{21}+Q_{22}\right) \geq 0, \\
1-\gamma c_{i}^{\prime} Q_{1} c_{i} \geq 0, i=1, \ldots, n_{f},
\end{gathered}
$$

where $\gamma=\sigma+\mathrm{e}^{2 \varphi d}$, and $\Phi(\cdot)=\left[\Phi_{i j}\right], i, j=1, \ldots, 6$, and $\Lambda(\cdot)=\left[\Lambda_{l k}\right], l, k=1, \ldots, 4$, are symmetric block matrices with nonzero blocks given by

$\Phi_{11}=\operatorname{He}\left\{\left[A_{a}(\delta) Q_{1}+R_{11}(x, \tilde{x}, \delta)\right] N\right\}+N^{\prime}\left(Q_{21}+Q_{22}\right) N$, $\Phi_{12}=B_{a}(\delta) F+N^{\prime} R_{21}(x, \tilde{x}, \delta)^{\prime}$

$$
+\left[R_{12}(x, \tilde{x}, \delta)-R_{11}(x, \tilde{x}, \delta)\right] N,
$$

$\Phi_{13}=N^{\prime} R_{31}(x, \tilde{x}, \delta)^{\prime}-R_{12}(x, \tilde{x}, \delta)$,

$\Phi_{14}=N^{\prime} R_{41}(x, \tilde{x}, \delta)^{\prime}-R_{11}(x, \tilde{x}, \delta)$,

$\Phi_{15}=N^{\prime} R_{51}(x, \tilde{x}, \delta)^{\prime}-R_{12}(x, \tilde{x}, \delta)$,

$\Phi_{16}=d N^{\prime} Q_{1} A_{a}(\delta)^{\prime}$,

$\Phi_{22}=\operatorname{He}\left\{\left[R_{22}(x, \tilde{x}, \delta)-R_{21}(x, \tilde{x}, \delta)\right] N\right\}-(1-h) N^{\prime} Q_{21} N$,

$\Phi_{23}=N^{\prime}\left[R_{32}(x, \tilde{x}, \delta)^{\prime}-R_{31}(x, \tilde{x}, \delta)^{\prime}\right]-R_{22}(x, \tilde{x}, \delta)$,

$$
\begin{aligned}
& \Phi_{24}=N^{\prime}\left[R_{42}(x, \tilde{x}, \delta)^{\prime}-R_{41}(x, \tilde{x}, \delta)^{\prime}\right]-R_{21}(x, \tilde{x}, \delta), \\
& \Phi_{25}=N^{\prime}\left[R_{52}(x, \tilde{x}, \delta)^{\prime}-R_{51}(x, \tilde{x}, \delta)^{\prime}\right]-R_{22}(x, \tilde{x}, \delta), \\
& \Phi_{26}=d F^{\prime} B_{a}(\delta)^{\prime}, \Phi_{33}=-Q_{22}-\operatorname{He}\left\{R_{32}(x, \tilde{x}, \delta)\right\}, \\
& \Phi_{34}=-R_{31}(x, \tilde{x}, \delta)-R_{42}(x, \tilde{x}, \delta)^{\prime}, \\
& \Phi_{35}=-R_{32}(x, \tilde{x}, \delta)-R_{52}(x, \tilde{x}, \delta)^{\prime}, \\
& \Phi_{44}=-Q_{3}-\operatorname{He}\left\{R_{41}(x, \tilde{x}, \delta)\right\}, \\
& \Phi_{45}=-R_{42}(x, \tilde{x}, \delta)-R_{51}(x, \tilde{x}, \delta)^{\prime}, \\
& \Phi_{55}=-Q_{3}-\operatorname{He}\left\{R_{52}(x, \tilde{x}, \delta)\right\}, \Phi_{66}=N^{\prime}\left(Q_{3}-2 Q_{1}\right) N, \\
& \Lambda_{11}=\operatorname{He}\left\{\left[A_{a}(\delta) Q_{1}+S_{1}(x, \delta)\right] N\right\} \\
& +N^{\prime}\left[Q_{21}+Q_{22}-2 \varphi Q_{1}\right] N, \\
& \Lambda_{12}=N^{\prime} S_{2}(x, \delta)^{\prime}-S_{1}(x, \delta), \Lambda_{13}=N^{\prime} S_{3}(x, \delta)^{\prime}-S_{1}(x, \delta), \\
& \Lambda_{14}=d N^{\prime} Q_{1} A_{a}(\delta)^{\prime} \text {, } \\
& \Lambda_{22}=-(1-h) Q_{21}-Q_{22}-\operatorname{He}\left\{S_{2}(x, \delta)\right\} \text {, } \\
& \Lambda_{23}=-S_{3}(x, \delta)^{\prime}-S_{2}(x, \delta), \Lambda_{33}=-Q_{3}-\operatorname{He}\left\{S_{3}(x, \delta)\right\} \text {, } \\
& \Lambda_{44}=N^{\prime}\left(Q_{3}-2 Q_{1}\right) N \text {, } \\
& N=\left[\begin{array}{ll}
I_{n} & 0_{n \times(m-n)}
\end{array}\right] \text {, } \\
& \Omega_{a}(x)=\left[\begin{array}{ll}
\Omega_{1}(x) & \Omega_{2}(x)
\end{array}\right], \\
& \Omega_{b}(x, \tilde{x})=\left[\begin{array}{cccccc}
\Omega_{a}(x) & 0 & 0 & 0 & 0 & 0 \\
0 & \Omega_{a}(\tilde{x}) & 0 & 0 & 0 & 0 \\
0 & 0 & 0 & 0 & 0 & \Omega_{a}(x)
\end{array}\right] \text {, } \\
& \Omega_{c}(x)=\left[\begin{array}{cccc}
\Omega_{a}(x) & 0 & 0 & 0 \\
0 & 0 & 0 & \Omega_{a}(x)
\end{array}\right] .
\end{aligned}
$$

Then, the origin of the closed-loop system given by (3) with

$$
K(\tilde{x})=F \Pi_{a}(\tilde{x}) Q_{1}^{-1}
$$

is robustly regionally stable, i.e., for any $\bar{x}_{0} \in \mathcal{R}_{0}$ and $\delta \in \Delta$, $x(t) \in \mathcal{R}$ for all $t \geq 0$ and $x(t) \rightarrow 0$ as $t \rightarrow \infty$, where $\mathcal{R}$ and $\mathcal{R}_{0}$ are as defined in (12) with $P_{1}=Q_{1}^{-1}$.

Proof: Suppose that (35)-(39) are satisfied. Then, by convexity arguments, (35), (36) and (37) are satisfied for all $(x, \tilde{x}, \delta) \in \mathcal{X} \times \mathcal{X} \times \Delta,(x, \delta) \in \mathcal{X} \times \Delta$ and $(x, \delta) \in \mathcal{X} \times \Delta$, respectively. In addition, let $P_{1}, P_{21}, P_{22}$ and $P_{3}$ be matrices satisfying the relations in (26) and observe that $\Omega_{a}(\cdot) \Pi_{a}(\cdot)=0$, $N \Pi_{a}(\cdot)=I_{n}$ and $K(\tilde{x}) Q_{1}=F \Pi_{a}(\tilde{x})$. In the following, it will be shown that the conditions of Lemma 4 hold.

Firstly, note from Lemma 1 that $Q_{3}-2 Q_{1} \geq-P_{3}^{-1}$, and thus (35) also holds with $\Phi_{66}$ replaced by $-N^{\prime} P_{3}^{-1} N$. Then, post- and pre-multiplying (35) with the latter $\Phi_{66}$ by $\operatorname{diag}\left\{\Pi_{a}(x), \Pi_{a}(\tilde{x}), I_{n}, I_{n}, I_{n}, \Pi_{a}(x)\right\}$ and its transpose, respectively, and applying Schur's complement to the resulting inequality leads to:

$$
\begin{array}{r}
\Psi(x, \tilde{x}, \delta)+\Upsilon(x, \tilde{x}, \delta)^{\prime} P_{3} \Upsilon(x, \tilde{x}, \delta)+\operatorname{He}\{R(x, \tilde{x}, \delta) \mathcal{I}\}<0, \\
\forall(x, \tilde{x}, \delta) \in \mathcal{X} \times \mathcal{X} \times \Delta, \quad(41)
\end{array}
$$

with $\Psi(\cdot), \Upsilon(\cdot)$ and $\mathcal{I}$ as defined previously and $R(x, \tilde{x}, \delta)$ is given by:

$$
R(x, \tilde{x}, \delta)=\left[\begin{array}{lllll}
R_{11}^{\prime} \Pi_{a}(x) & R_{21}^{\prime} \Pi_{a}(\tilde{x}) & R_{31}^{\prime} & R_{41}^{\prime} & R_{51}^{\prime} \\
R_{12}^{\prime} \Pi_{a}(x) & R_{22}^{\prime} \Pi_{a}(\tilde{x}) & R_{32}^{\prime} & R_{42}^{\prime} & R_{52}^{\prime}
\end{array}\right]^{\prime},
$$

where, for notation simplicity, $R_{i, j}$ denotes $R_{i, j}(x, \tilde{x}, \delta)$. Then, pre- and post-multiplying (41) by respectively $\eta\left(x_{t}\right)^{\prime}$ and $\eta\left(x_{t}\right)$ as in (24) and since $\mathcal{I} \eta\left(x_{t}\right) \equiv 0$, yields

$$
\begin{array}{r}
\eta\left(x_{t}\right)^{\prime}\left[\Psi(x, \tilde{x}, \delta)+\Upsilon(x, \tilde{x}, \delta)^{\prime} P_{3} \Upsilon(x, \tilde{x}, \delta)\right] \eta\left(x_{t}\right)<0, \\
\forall\left(x_{t}, \delta\right) \in \mathcal{X}_{a} \times \Delta, \eta\left(x_{t}\right) \not \equiv 0, t \geq t_{0} .
\end{array}
$$


Hence, taking (27) into account and noting that the inequality (42) is strict, $\mathcal{X}$ is bounded and $\eta\left(x_{t}\right)$ also depends on $x(t)$, there exists a sufficiently small scalar $\epsilon$ such that the condition $\mathbf{C 1}$ of Lemma 4 holds.

Now, pre- and post-multiplying (36) by $x(t)^{\prime} P_{1} \Pi_{a}(x)^{\prime}$ and $\Pi_{a}(x) P_{1} x(t)$, respectively, and considering the definition of $V_{1}(x(t))$ in (11) and the fact that

$$
\dot{x}(t)=f(x(t), \delta)=\Pi_{a}(x)^{\prime} A_{a}(\delta) x, \forall t \in\left[0, t_{0}\right),
$$

leads to:

$\dot{V}_{1}(x(t))-2 \varphi V_{1}(x(t)) \leq 0, \forall(x(t), \delta) \in \mathcal{X} \times \Delta, \forall t \in\left[0, t_{0}\right)$, and thus $\mathbf{C 2}$ holds.

Next, similar as for (35), by Lemma 1 it turns out that (37) also holds with $\Lambda_{44}$ replaced by $-N^{\prime} P_{3}^{-1} N$. Then, post- and pre-multiplying (37) with the latter $\Lambda_{44}$ by $\operatorname{diag}\left\{\Pi_{a}(x), I_{n}, I_{n}, \Pi_{a}(x)\right\}$ and its transpose, respectively, and applying Schur's complement to the resulting inequality leads to:

$$
\begin{array}{r}
\left.\widetilde{\Psi}(x, \delta)+\widetilde{\Upsilon}(x, \delta)^{\prime} P_{3} \widetilde{\Upsilon}(x, \delta)+\operatorname{He}\{S(x, \delta)\} \widetilde{\mathcal{I}}\right\} \leq 0, \\
\forall(x, \delta) \in \mathcal{X} \times \Delta,
\end{array}
$$

with $\widetilde{\Psi}(\cdot), \widetilde{\Upsilon}(\cdot)$ and $\widetilde{\mathcal{I}}$ as defined previously and $S(x, \delta)$ is given by:

$$
S(x, \delta)=\left[\begin{array}{lll}
S_{1}(x, \delta)^{\prime} \Pi_{a}(x) & S_{2}(x, \delta)^{\prime} & S_{3}(x, \delta)^{\prime}
\end{array}\right]^{\prime} .
$$

Pre- and post-multiplying (43) by respectively $\widetilde{\eta}\left(x_{t}\right)^{\prime}$ and $\widetilde{\eta}\left(t_{x}\right)$ given in (33) and considering (32) it follows that condition $\mathbf{C 3}$ holds.

Finally, taking the matrices defined in (26) into account, notice that (38) is equivalent to condition $\mathbf{C 4}$ whereas (39) ensures that the inclusion condition $\mathcal{R} \subset \mathcal{X}$ of $\mathbf{C 5}$ holds (see, e.g., [29]), which completes the proof.

Remark 3: Notice that if the inequalities of Theorem 1 are feasible for some $d=\bar{d}$ and $h=\bar{h}$, then they are also feasible for all $d<\bar{d}$ and $h<\bar{h}$. In addition, if there is a solution $u(\cdot)=K x(\cdot)$ of Theorem 1 for the linear approximation of system (1) around the system origin then there exists a solution of Theorem 1 for the original polynomial system and a sufficiently small region $\mathcal{X}$.

\section{A. Computational Issues}

Theorem 1 can be applied for designing the polynomial state feedback control law $u(\cdot)=K(x(\cdot)) x(\cdot)$, with $K(x(\cdot))$ as defined in (40), in order to regionally stabilize the closedloop system. Furthermore, the following design optimization problems are of interest.

Maximizing the set of admissible initial states: Firstly, assuming that the maximal admissible input delay $d$, its variation rate $h$ and the polytopic state-space domain $\mathcal{X}$ are given, the following optimization problem maximizes the volume of the set $\mathcal{R}_{0}$ of admissible initial states:

$$
\max _{\varphi, \sigma, Q_{1}, \ldots, F} \log \left(\operatorname{det}\left(Q_{1}\right)\right) \text { subject to (35)-(39). }
$$

If $\sigma$ and $\varphi$ are considered as free parameters, notice that the above optimization problem is non-convex and a direct numerical solution will be hard to be obtained. To derive a solution via semi-definite programming, it can be applied a gridding technique over $\sigma$ and $\varphi$ as considered in the numerical examples in Section V.
In addition, the shape and size of $\mathcal{X}$ play an important role in the maximization of $\mathcal{R}_{0}$, since it is implied by Theorem 1 that $\mathcal{R}_{0} \subseteq \mathcal{R} \subset \mathcal{X}$ to guarantee the positively invariance of $\mathcal{R}$. In practical applications, the shape and size of $\mathcal{X}$ may be estimated from the physical knowledge of state-space constraints or from directions where the system is likely to be initialized. When there is no previous knowledge on the physical constraints, the set $\mathcal{X}$ can be initially defined in terms of the following parameterized hyper-rectangle

$$
\mathcal{X}:=\left\{x \in \mathbb{R}^{n}:\left|x_{i}\right| \leq \mu_{i}, i=1, \ldots, n\right\},
$$

where $\mu_{i}, i=1, \ldots, n$, are positive scalars defining the size of $\mathcal{X}$. In this case, it can be straightforwardly applied the approach proposed in [30] for iteratively maximizing the sets $\mathcal{X}$ and $\mathcal{R}$.

Maximizing a lower bound on the maximum admissible delay: In order to maximize a lower bound on the maximum admissible delay $d$ ensuring the regional stability for a given set of initial states of the closed-loop system, it is assumed for simplicity that $h$ and $\mathcal{X}$ are given. In particular, the set of admissible initial states of interest is assumed to be an ellipsoid as described in the following:

$$
\widetilde{\mathcal{R}}_{0}:=\left\{x \in \mathbb{R}^{n}: x^{\prime} P_{0} x \leq 1\right\},
$$

where $P_{0}>0$ is a given matrix defining the size and shape of $\widetilde{\mathcal{R}}_{0}$. Thus, the following optimization problem can be formulated to maximize $d$ :

$\max _{d, \varphi, \sigma, Q_{1}, \ldots, F} d$ subject to (35)-(39) and $\left[\begin{array}{ll}P_{0} & I_{n} \\ I_{n} & Q_{1}\end{array}\right] \geq 0$.

Notice that the last inequality in (46) ensures that $\widetilde{\mathcal{R}}_{0} \subseteq \mathcal{R}_{0}$. In addition, similarly to the optimization problem of (44), a numerical solution in terms of LMI constraints can be obtained by means of a griding over $\sigma, \varphi$ and $d$.

\section{NumeriCAL EXAMPLES}

In this section, two numerical examples are provided to demonstrate the effectiveness of the proposed approach.

\section{A. Van der Pol equation}

Consider the following state-space representation of the Van der Pol equation with a delayed control input:

$$
\left\{\begin{array}{l}
\dot{x}_{1}(t)=x_{2}(t), \\
\dot{x}_{2}(t)=-x_{1}(t)+\delta\left(1-x_{1}^{2}(t)\right) x_{2}(t)+u(t-\tau(t)),
\end{array}\right.
$$

where $x=\left[\begin{array}{ll}x_{1} & x_{2}\end{array}\right]^{\prime} \in \mathbb{R}^{2}$ is the state, $\delta \in \mathbb{R}$ is a bounded uncertain constant parameter and $u \in \mathbb{R}$ is the control input being subject to a time-varying delay $\tau(t)$. Notice that the origin of the above system is open-loop unstable. In this example, it is assumed that the sets $\mathcal{X}$ and $\Delta$ defining respectively the state and uncertainty domains, as well as the maximum delay variation rate $h$ are known a priori and the following two scenarios will be considered: $(i)$ for a given maximum admissible delay $d$, determine a controller $u(t)=K(x) x(t)$ that maximizes the set of admissible initial states; and $(i i)$ for a given set of admissible initial states, determine a controller $u(t)=K(x) x(t)$ that maximizes a lower bound on the maximum admissible delay $d$.

To determine a polynomial matrix decomposition as in (19), observe that the largest polynomial degree of the system 
dynamics (47) with respect to $x$ is three. Then, the matrix $\Pi(x)$ has to contain the monomials in $x$ of degree one up to two. For instance, let $\Pi(x)$ be defined as below:

$$
\Pi(x)=\left[\begin{array}{ll}
x_{1} I_{2} & x_{1}^{2} I_{2}
\end{array}\right]^{\prime} .
$$

Then, the matrices $A_{a}(\delta)$ and $B(\delta)$ in (19) are as follows:

$$
\begin{aligned}
A_{a}(\delta) & =\left[\begin{array}{lll}
A_{0}^{\prime} & 0_{2} & A_{2}^{\prime}
\end{array}\right]^{\prime}, \\
B_{a}(\delta) & =\left[\begin{array}{lll}
B_{0}^{\prime} & 0_{1 \times 2} & 0_{1 \times 2}
\end{array}\right]^{\prime},
\end{aligned}
$$

where

$$
A_{0}=\left[\begin{array}{cc}
0 & 1 \\
-1 & \delta
\end{array}\right], A_{2}=\left[\begin{array}{cc}
0 & 0 \\
0 & -\delta
\end{array}\right], B_{0}=\left[\begin{array}{l}
0 \\
1
\end{array}\right],
$$

and the constraint in (20) is satisfied with the following matrices

$$
\Omega_{1}(x)=\left[\begin{array}{c}
x_{1} I_{2} \\
0_{2}
\end{array}\right], \Omega_{2}(x)=\left[\begin{array}{cc}
-I_{2} & 0_{2} \\
x_{1} I_{2} & -I_{2}
\end{array}\right] .
$$

Note that the matrix $\Omega_{2}(x)$ as above has full column-rank for all $x \in \mathbb{R}^{2}$.

It is considered that the maximum delay variation rate $h$ and the sets $\mathcal{X}$ and $\Delta$ are given by:

$$
\begin{gathered}
h=1, \mathcal{X}=\left\{x \in \mathbb{R}^{2}:\left|x_{i}\right| \leq \mu, i=1,2\right\}, \\
\Delta=\{\delta \in \mathbb{R}: 0.4 \leq \delta \leq 0.8\},
\end{gathered}
$$

where $\mu$ is a scalar defining the size of $\mathcal{X}$. Firstly, for $d=$ 0.1 , the optimization problem (44) to maximize $\mathcal{R}_{0}$ is solved considering the evaluation of inequalities (35)-(39) in a 2-D grid on $(\sigma, \varphi)$ and the iterative procedure of [30], leading to:

$$
\begin{gathered}
P_{1}=\left[\begin{array}{ll}
0.1096 & 0.0033 \\
0.0033 & 0.1096
\end{array}\right], \mu=3.3, \sigma=10^{-5}, \\
\varphi=0.880, K(x)=\left[\begin{array}{c}
0.0117-7.2315 \times 10^{-7} x_{1}^{2} \\
-1.7076+1.1461 \times 10^{-5} x_{1}^{2}
\end{array}\right]^{\prime} .
\end{gathered}
$$

Fig. 1 shows the obtained regions $\mathcal{R}_{0}$ and $\mathcal{R}$ (i.e., an estimate of the set of reachable states) as well as closed-loop trajectories starting at the boundary of $\mathcal{R}_{0}$ considering a constant delay $\tau(t)=0.1$ (solid black line) and a time-varying one $\tau(t)=$ $0.05(1+\cos (2 \pi t))$ (dashed black line).

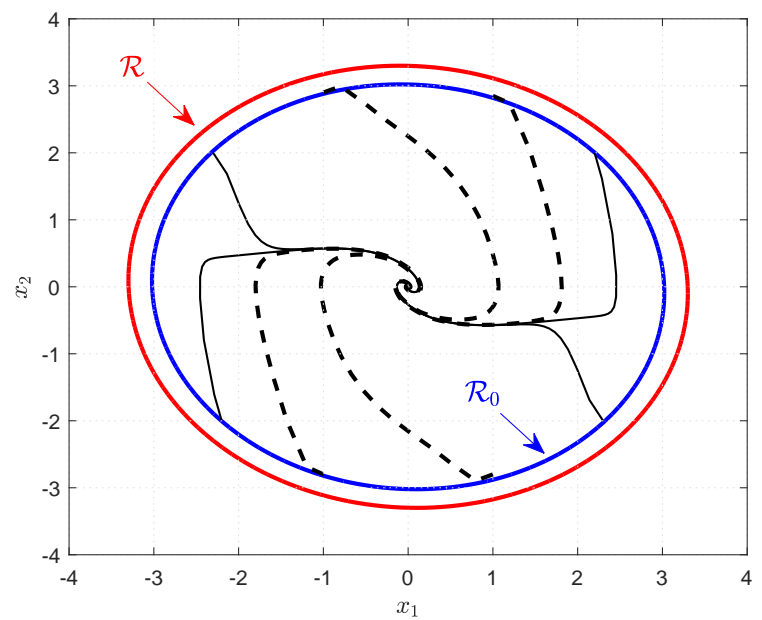

Figure 1. The sets $\mathcal{R}_{0}$ (blue line) and $\mathcal{R}$ (red line) and stable trajectories (solid and dashed black lines).

On the other hand, assuming that the set of admissible initial states is defined as in (45) with $P_{0}=2 I_{2}$, optimization problem (46) is considered to synthesize a controller in order to obtain a maximized lower bound $d$ on the admissible delay yielding:

$d=0.26, \varphi=2.218, K(x)=\left[\begin{array}{c}0.0803+4.1122 \times 10^{-6} x_{1}^{2} \\ -1.6411-5.9074 \times 10^{-5} x_{1}^{2}\end{array}\right]^{\prime}$, considering $\sigma=10^{-5}, \mu=1$ and a 2-D griding over $(\varphi, d)$.

\section{B. Event-based sampled-data polynomial system}

Consider the following open-loop unstable polynomial system taken from [23]:

$$
\left\{\begin{array}{l}
\dot{x}_{1}(t)=-1.5 x_{1}^{2}(t)-0.5 x_{1}^{3}(t)-x_{2}(t) \\
\dot{x}_{2}(t)=-u(t)
\end{array}\right.
$$

For the above system, it is considered that the control signal $u(t)$ is an event-based sampled-data state feedback control law with a bounded sampling interval, i.e., $u(t)=u\left(t_{k}\right)$, $t \in\left[t_{k}, t_{k+1}\right), t_{k+1}-t_{k} \leq d, \forall k \in \mathbb{Z}^{+}$. In this setting, it is assumed that the state $x=\left[\begin{array}{ll}x_{1} & x_{2}\end{array}\right]^{\prime}$ is sampled at time instants $t_{0}, t_{1}, \ldots, t_{k}, \ldots$ satisfying $0<t_{0}<t_{1}<\cdots<t_{k}<$ $\cdots, \forall k \in \mathbb{Z}^{+}$. In this example, it is aimed to design a locally stabilizing controller that maximizes $d$ assuming that the first sampling instant $t_{0}$ can take any value in the interval $(0, d]$. Following the approach in [31] with a zero transmission delay, the aperiodic sampled-data control signal can be modeled as a time-delayed signal with a bounded varying delay satisfying $\tau(t):=t-t_{k} \leq d, \dot{\tau}(t)=1, \forall t \in\left[t_{k}, t_{k+1}\right)$, and being reset to zero at the sampling instants.

Similarly to Example V-A, a polynomial decomposition of the system dynamics can be obtained by considering the matrices $\Pi(x)$ together with $\Omega_{1}(x)$ and $\Omega_{2}(x)$ as in (48) and (51), respectively, and with $A_{a}=\left[\begin{array}{lll}A_{0}^{\prime} & A_{1}^{\prime} & A_{2}^{\prime}\end{array}\right]^{\prime}$,

$$
\begin{gathered}
B_{a}=\left[\begin{array}{lll}
B_{0}^{\prime} & 0_{1 \times 2} & 0_{1 \times 2}
\end{array}\right]^{\prime}, B_{0}=\left[\begin{array}{ll}
0 & -1
\end{array}\right]^{\prime}, \\
A_{0}=\left[\begin{array}{cc}
0 & -1 \\
0 & 0
\end{array}\right], \quad A_{1}=\left[\begin{array}{cc}
-1.5 & 0 \\
0 & 0
\end{array}\right], A_{2}=\left[\begin{array}{cc}
-0.5 & 0 \\
0 & 0
\end{array}\right] .
\end{gathered}
$$

In this example, it is considered that the state-space domain and the set of admissible initial states are respectively given by $\mathcal{X}=\left\{x \in \mathbb{R}^{2}:\left|x_{i}\right| \leq 0.6, i=1,2\right\}$ and $\widetilde{\mathcal{R}}_{0}=\{x \in$ $\left.\mathbb{R}^{2}: x_{1}^{2}+x_{2}^{2} \leq 0.25^{2}\right\}$. Then, the optimization problem (46) is applied to maximize $d$ such that the origin of the closedloop system is regionally stable leading to $d=0.112$, i.e., $t_{k+1}-t_{k} \leq 0.112$, for all $k \in \mathbb{Z}_{+}$, and

$P_{1}=\left[\begin{array}{cc}4.3912 & -1.4268 \\ -1.4268 & 4.2385\end{array}\right], K(x)=\left[\begin{array}{c}-1.5420-0.0057 x_{1}^{2} \\ 1.6718+0.0052 x_{1}^{2}\end{array}\right]^{\prime}$,

considering $\sigma=10^{-5}$ and $\varphi=0.890$. It should be noted that in [23] a locally stabilizing controller has been designed for a maximum sampling interval $d=0.099$ [sec] and it is not guaranteed that a controller can be designed for $d \geq 0.1$. Furthermore, [23] does not provide a domain of stability. The regions $\mathcal{R}_{0}$ and $\mathcal{R}$ are shown in Fig. 2 as well as stable (black dashed line) and unstable (black dotted line) closed-loop trajectories starting close at the boundary of $\mathcal{R}_{0}$ considering a constant sampling interval of 0.112 (i.e., $t_{k+1}-t_{k}=0.112$, $\forall k \in \mathbb{Z}^{+}$) with $t_{0}=0.112$. In particular, it can be observed that there exist divergent trajectories starting very close to the computed domain of stability, which shows that the method can provide good estimates of the region of attraction. 


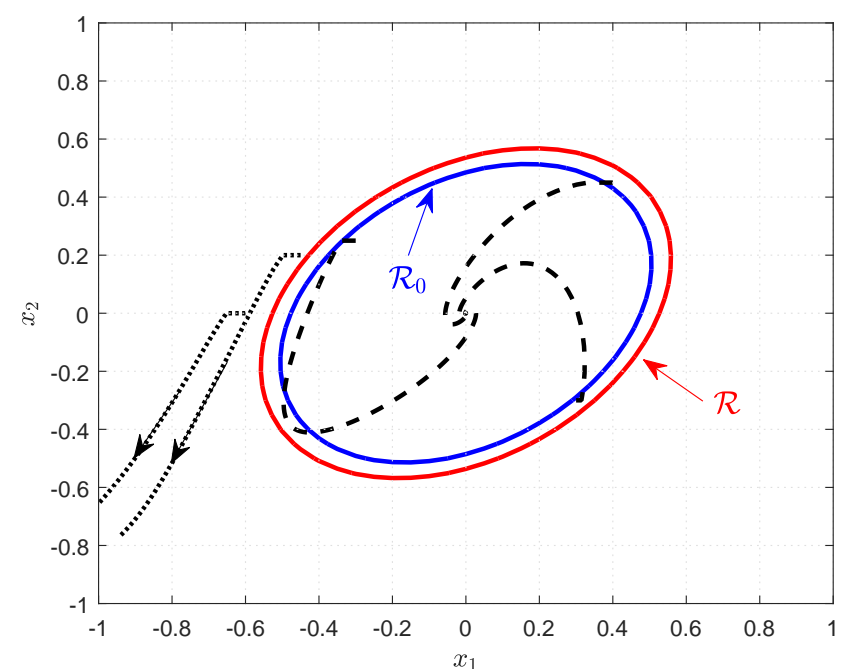

Figure 2. The sets $\mathcal{R}_{0}$ (blue line) and $\mathcal{R}$ (red line), as well as stable (dashed black lines) and unstable (dotted black lines) trajectories.

\section{CONCLUDING REMARKS}

This paper has addressed the problem of regional stabilization of open-loop unstable input-delayed nonlinear polynomial systems with polytopic parameter uncertainty. Considering a novel polynomial decomposition of the system dynamics, the control synthesis is cast in terms of LMIs and is based on the Lyapunov-Krasovskii stability theory. In particular, the proposed conditions take into account that the system initially operates in open-loop for a certain interval of time due to the input delay. Then, a nonlinear polynomial state-feedback controller is designed such that the zero equilibrium solution of the closed-loop system under a bounded time-varying input delay is robustly locally uniformly asymptotically stable with a guaranteed stability region. Two optimization procedures have also been proposed to either maximize the set of admissible initial states (assuming a given bound on the input delay and its variation rate) or maximize a lower bound on the maximum input delay for a given set of admissible initial states. Numerical examples have demonstrated the potentials of the proposed approach.

\section{REFERENCES}

[1] D. Yue and Q.-L. Han, "Delayed feedback control of uncertain systems with time-varying input delay," Automatica, vol. 41, pp. 233-240, 2005.

[2] F. Mazenc, S.-I. Niculescu, and M. Krstic, "Lyapunov-Krasovskii functionals and application to input delay compensation for linear timeinvariant systems," Automatica, vol. 48, pp. 1317-1323, 2012.

[3] W. Zhang, M. S. Branicky, and S. M. Phillips, "Stability of networked control systems stability of networked control systems," IEEE Control Systems Magazine, vol. 21, no. 1, pp. 84-99, 2001.

[4] W. P. M. H. Heemels, A. R. Teel, N. van de Wouw, and D. Nesic, "Networked control systems with communication constraints: Tradeoffs between transmission intervals, delays and performance," IEEE Transactions on Automatic Control, vol. 55, no. 8, pp. 1781-1796, 2010.

[5] L. Mirkin and N. Raskin, "Every stabilizing dead-time controller has an observer-predictor-based structure," Automatica, vol. 39, pp. 1747-1754, 2003.

[6] Z. Aststein, "Linear Systems with Delayed Controls: A Reduction," IEEE Transactions on Automatic Control, vol. 27, no. 4, pp. 869-879, 1982.

[7] K. Liu and E. Fridman, "Delay-dependent methods and the first delay interval," Systems \& Control Letters, vol. 64, pp. 57-63, 2014.
[8] R. Sanz, P. Garcia, P. Albertos, and Q.-C. Zhong, "Robust controller design for input-delayed systems using predictive feedback and an uncertainty estimator," International Journal of Robust and Nonlinear Control, vol. 27, no. 10, pp. 1826-1840, 2017.

[9] F. Mazenc, M. Malisoff, and Z. Lin, "Further results on input-to-state stability for nonlinear systems with delayed feedbacks," Automatica, vol. 44, pp. 2415-2421, 2008.

[10] H.-L. Choi and J.-T. Lim, "Asymptotic stabilization of an input-delayed chain of integrators with nonlinearity," Systems \& Control Letters, vol. 59, pp. 374-379, 2010.

[11] N. Fischer, R. Kamalapurkar, N. Fitz-Coy, and W. E. Dixon, "Lyapunovbased control of an uncertain Euler-Lagrange system with time-varying input delay," in Proocedings of the 2012 American Control Conference, Montreal, Canada, Jun 2012, pp. 3919-3924.

[12] Y.-E. Wang, X.-M. Sun, Z. Wang, and J. Zhao, "Construction of Lyapunov-Krasovskii functionals for switched nonlinear systems with input delay," Automatica, vol. 50, pp. 1249-1253, 2014.

[13] M. Krstic, "Input delay compensation for forward complete and strictfeedforward nonlinear systems," IEEE Transactions on Automatic Control, vol. 55 , no. 2 , pp. 287-303, 2010 .

[14] B. Arguello-Serrano and M. Velez-Reyes, "Nonlinear control of a heating, ventilating, and air conditioning system with thermal load estimation," IEEE Transactions on Control Systems Technology, vol. 7, no. 1 , pp. 56-63, 1999.

[15] P. Thounthong and S. Pierfederici, "A new control law based on the differential flatness principle for multiphase interleaved DC-DC converter," IEEE Transactions on Circuits and Systems II: Express Briefs, vol. 57, no. 11, pp. 903-907, 2010.

[16] Z. Yang and H. Sun, "Nonlinear control design of a hypersonic aircraft using sum-of-squares method," in Informatics in Control, Automation and Robotics, D. Yang, Ed. Berlin: Springer, 2012, pp. 333-342.

[17] A. Tayebi and S. McGilvray, "Attitude stabilization of a VTOL quadrotor aircraft," IEEE Transactions on Control Systems Technology, vol. 14, no. 3, pp. 562-571, 2006.

[18] A. Papachristodoulou and S. Prajna, "A tutorial on sum of squares techniques for systems analysis," in Proceedings of the 2005 American Control Conference, Portland, OR, 2005, pp. 2686-2700.

[19] G. Chesi, "Computing Output Feedback Controllers to Enlarge the Domain of Attraction in Polynomial Systems," IEEE Transactions on Automatic Control, vol. 49, no. 10, pp. 1846-1850, 2004.

[20] L. El Ghaoui and G. Scorletti, "Control of rational systems using linearfractional representations and linear matrix inequalities," Automatica, vol. 32, no. 9, pp. 1273-1284, 1996.

[21] D. Coutinho, C. E. de Souza, and A. Trofino, "Stability analysis of implicit polynomial systems," IEEE Transactions on Automatic Control, vol. 54, no. 5, pp. 1012-1028, 2009.

[22] D. Coutinho and C. E. de Souza, "Delay-dependent robust stability and $L_{2}$-gain analysis of a class of nonlinear time-delay systems," Automatica, vol. 44, no. 8, pp. 2006-2018, 2008.

[23] H. S. Kim, J. B. Park, and Y. H. Joo, "Input-delay approach to sampleddata $\mathcal{H}_{\infty}$ control of polynomial systems based on a sum-of-squares analysis," IET Control Theory \& Applications, vol. 11, no. 9, pp. 14741484, 2017.

[24] A. Papachristodoulou, M. M. Peet, and S. Lall, "Analysis of polynomial systems with time delays via the sum of squares decomposition," IEEE Transactions on Automatic Control, vol. 54, no. 5, pp. 1058-1064, 2009.

[25] M. C. de Oliveira, J. C. Geromel, and J. Bernussou, "Extended $H_{2}$ and $H_{\infty}$ norm characterizations and controller parametrizations for discretetime systems," International Journal of Control, vol. 75, no. 9, pp. 666679, 2002.

[26] K. Gu, V. L. Kharitonov, and J. Chen, Stability of Time-Delay Systems. Boston, MA: Birkhäser, 2003.

[27] J. Hale and S. Lunel, Introduction to Functional Differential Equations. New York: Springer-Verlag, 1993.

[28] H. K. Khalil, Nonlinear Systems, 2nd ed. Upper Saddle River, NJ: Prentice-Hall, 1996.

[29] S. Boyd, L. El Ghaoui, E. Feron, and V. Balakrishnan, Linear Matrix Inequalities in System and Control Theory. Philadelphia, PA: SIAM, 1994.

[30] D. Coutinho and C. E. de Souza, "Nonlinear state feedback design with a guaranteed stability domain for locally stabilizable unstable quadratic systems," IEEE Transactions on Circuits and Systems I: Regular Papers, vol. 59, no. 2, pp. 360-370, 2012.

[31] I. F. M. Ghiggi, J. M. Gomes da Silva Jr., D. Coutinho, and C. E. de Souza, "Stability analysis of nonlinear rational sampled-data control systems over communication networks," in Proceedings of the 2015 European Control Conference, Linz, Austria, 2015, pp. 422-427. 